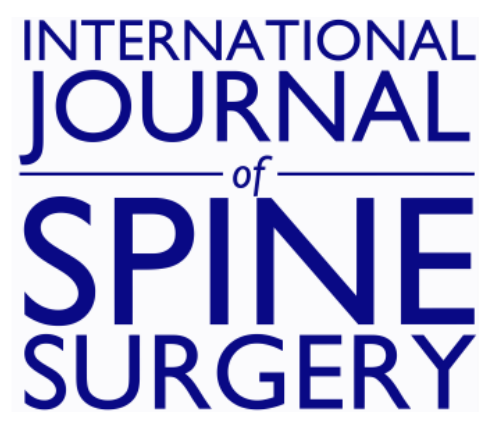

\title{
Safety and Efficacy of Bioabsorbable Cervical Spacers and Low-Dose rhBMP-2 in Multi-Level ACDF
}

Kaveh Khajavi and Alessandria Shen

Int J Spine Surg 2014, 8 ()

doi: https://doi.org/10.14444/1009

http://ijssurgery.com/content/8/9

This information is current as of April 26, 2023.

Email Alerts Receive free email-alerts when new articles cite this article. Sign up at:

http://ijssurgery.com/alerts

The International Journal of Spine Surgery

2397 Waterbury Circle, Suite 1,

Aurora, IL 60504, Phone: +1-630-375-1432

(C) 2014 ISASS. All Rightss Rifseryed 


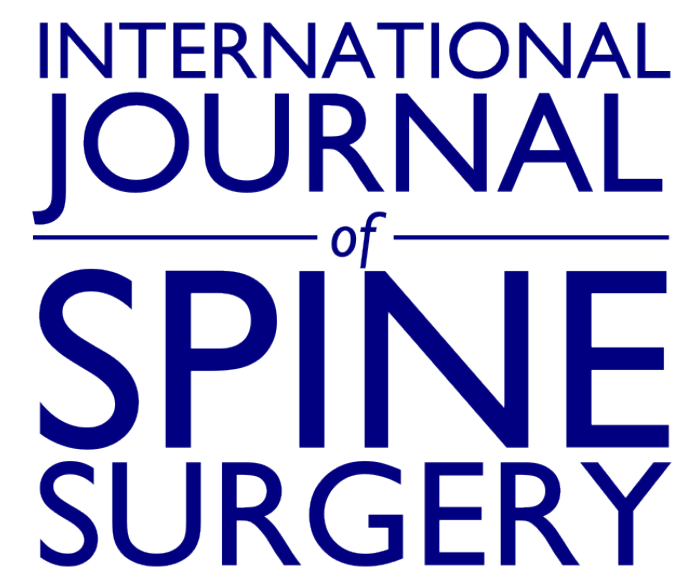

This article generously published free of charge by the International Society for the Advancement of Spine Surgery.

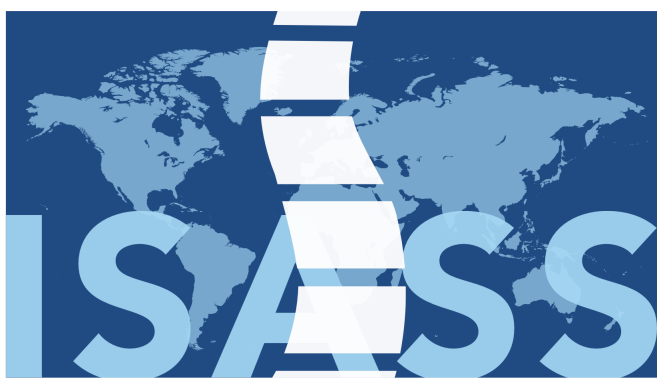

INTERNATIONAL SOCIETY for the ADVANCEMENT of SPINE SURGERY 


\section{Safety and Efficacy of \\ Bioabsorbable Cervical Spacers \\ and Low-Dose rhBMP-2 in \\ Multi-Level ACDF}

Kaveh Khajavi, MD, Alessandria Shen, MSPH

Georgia Spine and Neurosurgery Center

\section{Abstract}

\section{Introduction}

Many options for interbody spacer and graft biologic exist for multilevel anterior cervical discectomy

and fusion (ACDF). The objective of this study is to evaluate the safety and efficacy of a bioabsorbable cervical spacer (BCS) (Cornerstone HSR, Medtronic Sofamor Danek) filled with low-dose rhBMP-2 (INFUSE, Medtronic Sofamor Danek) in multilevel ACDF.

\section{Methods}

72 consecutive patients treated with a multi-level ACDF using BCS and rhBMP-2 (dosage between 0.5 to $0.7 \mathrm{mg}$ per level) at a single institution were followed in an IRBapproved, prospective registry. A total of 187 levels were treated $($ mean $=2.6)$, with 37 (51\%) patients undergoing a 2-level procedure and 35 (49\%) undergoing a 3- or 4-level procedure. Statistical analysis included frequency and ANOVA tests. Significance was accepted for $\mathrm{p}<0.001$.

\section{Results}

Average follow-up was 13.8 months. Mean patient age was 55.3 years, $70.8 \%$ were female, and $16.7 \%$ had undergone a previous cervical procedure. $29(40 \%)$ patients had cervical spondylitic myelopathy, 27 (38\%) had radiculopathy, 15 (21\%) had a combination of both, and $1(1 \%)$ patient had a previous nonunion.

A total of 187 levels were treated with an ACDF, with 37 (51\%) 2-level, 27 (38\%) 3-level, and 8 (11\%) 4-level cases. Average OR time, EBL, and LOS were 144 minutes, $49 \mathrm{~mL}$, and 1.1 days, respectively. Major complications occurred in 5 (7\%) patients: 2 returns to OR (1 nonunion, 1 seroma), 1 recurrent laryngeal nerve injury, and 2 hospital readmissions for excessive pre-vertebral swelling/dysphagia treated with steroids and 
observation. Minor complications occurred in 3 (4\%) patients: 2 exacerbations of preexisting medical conditions ( 1 atrial fibrillation, 1 COPD), and 1 hospital readmission for nausea/ headache due to narcotics.

At last follow-up, NDI improved $43 \%$ from $43.6 \%$ to $25.0 \%$. VAS neck pain improved $60 \%$ from 5.5 to 2.2 and VAS arm pain improved 52\% from 5.8 to 2.6. SF-36 PCS improved $24 \%$ from 37.5 to 46.3 and MCS improved $18 \%$ from 43.2 to 50.9. All clinical improvements were statistically significant $(\mathrm{p}<0.001)$.

Patient satisfaction was high, with $97 \%$ of patients reported being satisfied with their surgical outcome, and $90 \%$ would undergo the procedure again.

\section{Conclusion}

Patients in this series experienced significant clinical improvements, low complication rates, and high patient satisfaction. Symptomatic pseudoarthrosis was rare. BCS filled with low-dose rhBMP-2 appears to be a safe and effective option in multilevel ACDF. Further investigation is warranted.

keywords: $A C D F$, bioabsorbable, spacer, Outcomes, safety

Volume 8 Article 9 doi: 10.14444/1009

\section{Introduction}

Anterior cervical discectomy and fusion (ACDF) is a well-accepted treatment for symptomatic cervical spondylosis and degenerative disc disease (DDD). For single-level ACDFs, fusions rates are typically high, regardless of graft choice or the use of an anterior cervical plate. ${ }^{1,2,3,4,5,6}$ However, for multi-level (two or more levels) ACDFs, clinical efficacy can vary substantially, with fusion rates being reported to be between $56 \%$ and $100 \%$, depending on a variety of factors including the choice of interbody spacer, supplemental instrumentation, and graft biologic. $1,7,8,9$

Graft options have traditionally been limited to autologous structural iliac crest bone graft (ICBG) or allograft. Structural ICBG tends to yield higher fusion rates compared to

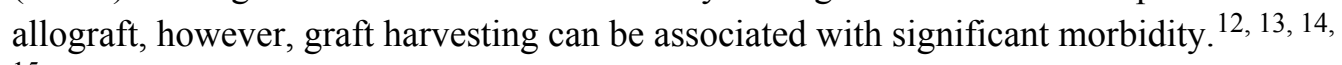

${ }^{15}$ Allograft bone offers many advantages, but tends to have lower fusion rates in multilevel ACDFs. ${ }^{16}$

One alternative spacer option is the Cornerstone HSR (Medtronic Sofamor Danek, Memphis, TN) bioabsorbable cervical spacer (BCS). The nature of this BCS allows it to be rigid during implantation to provide immediate biomechanical stability, but steadily degrades over time, allowing gradual transfer of stresses to the graft material. ${ }^{1,23}$ These spacers offer the advantages of being radiolucent, which facilitates fusion assessment, and have a modulus of elasticity similar to bone, which potentially decreases the incidence of subsidence, collapse, and stress shielding. ${ }^{1,23}$

While the use of these BCS has been studied in animal models and several retrospective studies, the choice of graft biologic has largely been limited to allograft or autograft materials. Recombinant human bone morphogenic protein-2 (rhBMP-2) (INFUSE®, 
Medtronic Sofamor Danek, Memphis, TN) is one alternative option, but has received much controversy in recent years due to its potential for high complication rates. ${ }^{10,11}$ However, we hypothesize that in low doses and in combination with BCS, rhBMP-2 can be beneficial in aiding high fusion rates in multi-level ACDF with acceptable morbidity rates. The purpose of this study is to evaluate the safety and efficacy of BCS filled with low-dose rhBMP-2 in multi-level ACDF.

\section{Methods}

A total of 72 consecutive patients who were treated with a multi-level ACDF using BCS and rhBMP-2 at a single institution between 2007 and 2012 were identified and included for analysis. Data were collected prospectively as part of an IRB-approved prospective registry. Demographic, treatment, and complication variables were collected through chart review. Outcome variables were collected prospectively and included disability (Neck Disability Index [NDI]), neck and arm pain (numeric rating scale [NRS], and quality of life (SF-36 physical and mental component scores [PCS \& MCS]) measures. Clinical outcomes and patient satisfaction were collected at preoperative and prospectively at $1,3,6,12$, and 24 months postoperative.

All patients had initial x-rays within 24 hours of surgery, AP and lateral x-rays at 4 to 6 weeks postoperative, and serial x-rays with flexion and extension views at 3, 6, 12, and 24 months. Fusion was defined as a lack of instrumentation failure, no lucency in the intervertebral disc space, and no movement on flexion-extension radiographs ( $<2 \mathrm{~mm}$ of spinous process splaying was allowed). If there was uncertainty regarding fusion status, a CT scan of the cervical spine was obtained for confirmation, but otherwise was not routinely used.

Mean follow-up of the 72 patients was 13.8 months (range 6-24 months). Average patient age at time of surgery was 55.3 years (range $37-82$ ) and $71 \%$ were female. Average BMI was $28.4 \mathrm{~kg} / \mathrm{m}^{2}$ (range 18.5-41.2), 33\% were smokers, and 17\% had previous cervical surgery (laminectomy, fusion, or both). Primary clinical indication for surgery was classified as cervical spondylitic myelopathy (CSM) in 29 (40\%) patients, as radiculopathy (RAD) in 27 (38\%) patients, as a combination of CSM and RAD in 15 $(21 \%)$ patients, and non-union in $1(1 \%)$ patient. Complete demographic information is included in Table 1.

Table 1. Patient demographic and treatment information.

\begin{tabular}{|c|c|c|c|}
\hline & 2 levels $(n=37)$ & $3+$ levels $(n=35)$ & All $(\mathrm{N}=72)$ \\
\hline Age (years) mean - mean $\pm S D$ & $51.8 \pm 9.5$ & $59.0 \pm 10.2$ & $55.3 \pm 10.4$ \\
\hline Female - n (\%) & $26(70.3)$ & $25(71.4)$ & $51(70.8)$ \\
\hline BMI $\left(\mathbf{k g} / \mathbf{m}^{2}\right)-$ mean \pm SD & $27.9 \pm 5.6$ & $28.8 \pm 5.7$ & $28.4 \pm 5.6$ \\
\hline Tobacco Use - n (\%) & $16(43.2)$ & $8(22.9)$ & $24(33.3)$ \\
\hline \multicolumn{4}{|l|}{ Co-Morbidities Type - n (\%) } \\
\hline Diabetes & $5(13.5)$ & $6(17.1)$ & $11(15.3)$ \\
\hline
\end{tabular}




\begin{tabular}{|c|c|c|c|c|}
\hline Depression & $8(21.6)$ & $3(8.6)$ & $11(15.3)$ & \\
\hline Previous Surgery - n (\%) & $8(21.6)$ & $4(11.4)$ & $12(16.7)$ & \\
\hline Number of Levels - mean $\pm \mathrm{SD}$ & $2.0 \pm 0.0$ & $3.23 \pm 0.4$ & $2.59 \pm 0.7$ & \\
\hline OR Time (min) - mean \pm SD & $123.3 \pm 33.0$ & $171.3 \pm 36.4$ & $144.4 \pm 41.8$ & \\
\hline $\mathbf{E B L}(\mathbf{m L})-$ mean $\pm \mathrm{SD}$ & $52.9 \pm 57.0$ & $44.6 \pm 20.0$ & $49.0 \pm 43.8$ & \\
\hline LOS (days) - mean $\pm \mathrm{SD}$ & $1.2 \pm 0.8$ & $1.0 \pm 0.0$ & $1.1 \pm 0.6$ & \\
\hline
\end{tabular}

A total of 187 levels were treated with an ACDF, with 37 (51\%) 2-level, 27 (38\%)

3-level, and 8 (11\%) 4-level cases. Twenty (28\%) patients were treated at C3-4, 51 (71\%) patients were treated at C4-5, $65(90 \%)$ patients were treated at C5-6, and $50(69 \%)$ patients were treated at the C6-7 level. Bioabsorbable cervical spacers (Cornerstone HSR, Medtronic Sofamor Danek, Memphis, TN) were used, supplemented with rhBMP-2 (Infuse ${ }^{\circledR}$, Medtronic Sofamor Danek, Memphis, TN) osteoinductive graft material. Dosage for the rhBMP-2 was $0.5 \mathrm{mg}$ per level in $43(60 \%)$ patients and $0.7 \mathrm{mg}$ per level in $29(40 \%)$, for an average of $0.6 \mathrm{mg}$ was used at each level. A higher dosage of $0.7 \mathrm{mg}$ was initially used as routine practice, however after reports of possible complications associated the use of rhBMP-2 in the cervical spine surfaced, a lower dosage of $0.5 \mathrm{mg}$ was used routinely, instead. Monitoring in all cases included somatosensory evoked potentials (SSEP), motor evoked potentials (MEP), and upper extremity electromyographies (EMG). Additional anterior cervical plates (Atlantis, Medtronic Sofamor Danek, Memphis, TN) were also used in all cases. Postoperative collars were not prescribed for patients treated at 2 levels, were occasionally utilized for patients treated at 3 levels, and were most commonly prescribed for patients treated at 4 levels.

Statistical analyses included frequency analysis to examine demographic and treatment variables and one-way ANOVA to examine changes in clinical outcomes from pre- to all postoperative time points. All analyses were performed using SAS v9.3 (SAS Institute, Cary, NC) and statistical significance was accepted at the 0.05 level.

\section{Results}

Overall mean operative time (ORT), estimated blood loss (EBL), and postoperative length of stay (LOS) were 144 minutes, $49 \mathrm{~mL}$, and 1.1 days, respectively. There were no intraoperative complications. Although postoperative dysphagia was a common sideeffect of ACDF immediately after surgery, 38 (53\%) patients complained of persistent dysphagia beyond the first postoperative visit ( 2 weeks), $55 \%$ of which resolved by 1 month, $34 \%$ by 3 months, and $5 \%$ by 6 months, all without additional intervention. Two of the 38 patients were readmitted for observation and steroid administration due to worsening dysphagia and/or excessive prevertebral swelling (admission primarily for airway concerns). Each of these two patients received $0.5 \mathrm{mg}$ rhBMP-2 at each level treated. One patient was treated at four levels and the other was treated at two levels. Major complications occurred in 3 (4\%) patients, and included 2 returns to OR ( 1 nonunion revision, 1 retropharyngeal seroma evacuation) and 1 recurrent laryngeal nerve injury. Minor complications occurred in 5 (7\%) patients, and included the two aforementioned hospital readmissions for excessive pre-vertebral swelling/dysphagia, 1 hospital readmission within 30 days for nausea/headache due to prescribed narcotic-use, 
and 2 exacerbations of pre-existing medical conditions (1 COPD, 1 atrial fibrillation). There were no cases of infection or wound problems, hematoma formation, or new postoperative neurologic deficit. Overall fusion was $98.6 \%$ of patients or $99.5 \%$ of treated levels.

From preoperative to 12 months postoperative, NDI improved $43 \%$ (range $6-78 \%$, SD $17 \%$ ) from $44 \%$ to $25 \%$. NRS neck pain improved $60 \%$ (range $0-100 \%$, SD $14 \%$ ) from 5.5 to 2.2 and NRS arm pain improved $55 \%$ (range $0-100 \%$, SD 13\%) from 5.8 to 2.6. SF-36 PCS improved 24\% from 37.5 to 46.3 and MCS improved 18\% from 43.2 to 50.9 . All clinical improvements were statistically significant $(p<0.001)$. Complete clinical outcomes are included in Figure 1 and Figure 2.

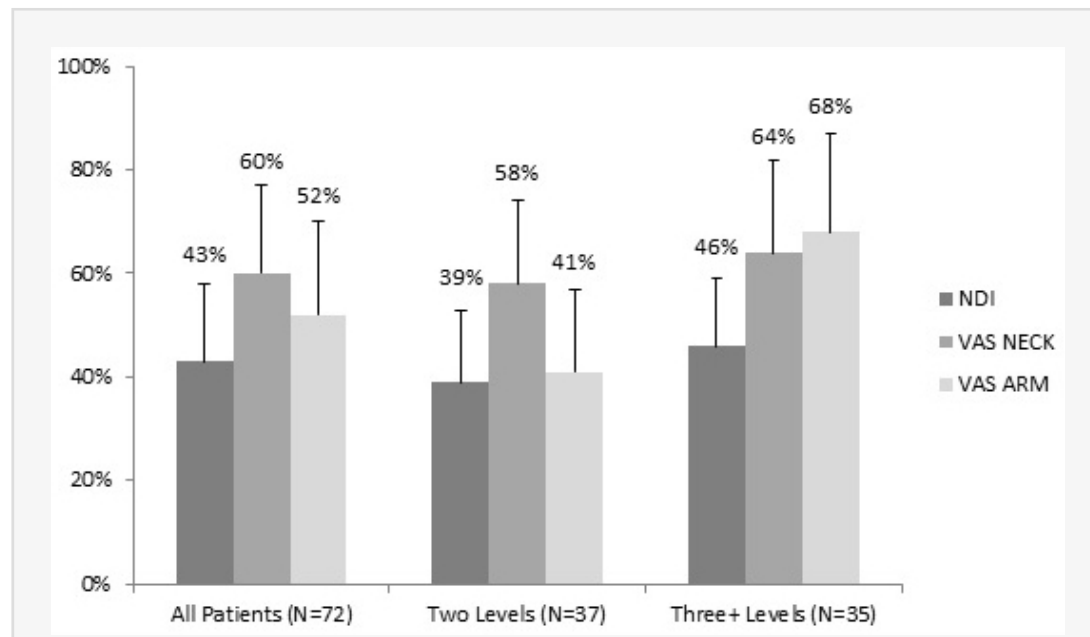

Fig. 1. Percentage improvements in disability and pain, stratified by levels treated.

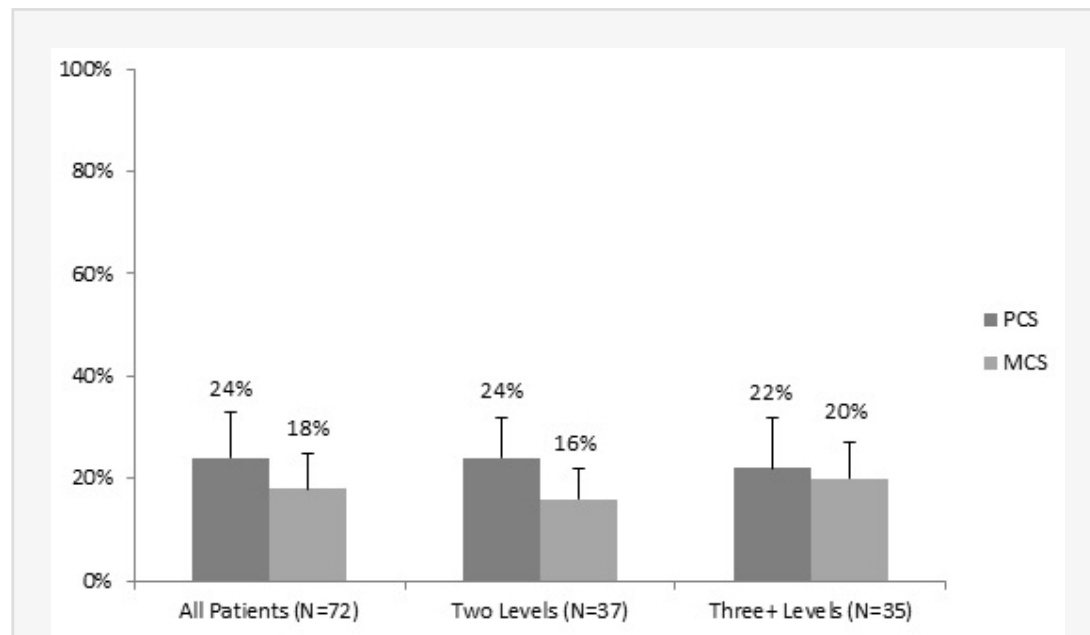

Fig. 2. Percentage improvements in physical and mental QOL, stratified by levels treated.

At last follow-up, 97\% of patients reported being either "satisfied" or "very satisfied" with their surgical outcome, and $90 \%$ indicated they would choose the same procedure again, given their outcome.

\section{Discussion}


Among the various available grafting options for multilevel ACDFs, autologous bone from the iliac crest has traditionally been considered the gold standard. 1, 2, 12, 13, 14, 15 Harvesting of iliac crest autograft can be associated with potential complications such as hematoma, surgical site infection, lateral femoral cutaneous nerve injury, pelvic fracture, muscle herniation, and persistent pain, and as a result, is becoming less commonly used today. ${ }^{2}$ Allograft is often used as an alternative to autologous bone, however, there may be an increased likelihood of failed fusion and need for additional revision surgery. ${ }^{16} \mathrm{In}$ order to avoid donor morbidity, synthetic spacers filled with a graft biologic have become an increasingly popular alternative. One commonly used option for interbody spacers are polyetheretherketone (PEEK) spacers, which offer the advantages of radiolucency and an elastic modulus closer to cortical bone. ${ }^{17,18,19}$ However, there exist concerns about particulate debris, as well as the limited amount of graft biologic that can be used due to their small apertures (relative to the cross-sectional endplate) and high costs. ${ }^{17,18,19}$ Titanium cages are also commonly used, but have the additional disadvantages of having a modulus of elasticity much higher than cortical bone, and their lack of radiolucency make fusion assessment difficult. ${ }^{20,21}$

Given the disadvantages of PEEK and titanium spacers, intervertebral spacers derived from bioabsorbable materials have emerged as a promising alternative. In addition to its radiolucency property and modulus of elasticity similar to bone, bioabsorbable implants degrade slowly over time (maintains 90\% strength until 6 months after implantation, degrades to $50 \%$ strength by 12 months postoperative), resulting in a gradual "dynamization" across the implant, graft material, and vertebral body endplates, all of which may increase the likelihood of a solid fusion. Since BCS gradually resorb, longterm problems associated with stress shielding, particulate debris, and retained foreign body are significantly minimized. ${ }^{1,23}$

While high fusion rates and clinical efficacy has been widely reported for single-level cases, there is a much wider discrepancy regarding the safety and utility of ACDF in multi-level cases. ${ }^{25}$ The reported fusion rates and complications rates for these procedures can vary widely and are highly dependent upon the number of levels treated, the use of supplemental instrumentation, and the choice of graft biologic and interbody spacer. ${ }^{1,2,3}$, $4,5,6,7,8,9$ Pseudoarthrosis rates of $0 \%$ to $44 \%$ have been reported, however, these numbers may be confounded by the lack of standardized fusion assessment and definition. 1, 24, 25 In general, fusion rates are thought to decrease with more levels treated, the use of allograft biologic, and a lack of rigid instrumentation. Complication rates also vary widely, between $2 \%$ to $67 \%$, particularly in regards to commonly-reported approachrelated events such as dysphagia, and also generally increase with the number of levels treated and potentially the source of graft. $^{24}$

Although short-term dysphagia is recognized as a ubiquitous and well-known side effect following $\mathrm{ACDF}$, there is nonetheless a considerable variability in the reported literature regarding incidence and prevalence rates. ${ }^{24,25,26}$ This variability can largely be attributed to discrepancies in both the definition and assessment of dysphagia. The qualitative assessment of dysphagia used in the present study defined "swallowing impairment" to include difficulty with solid and/or liquid foods and pain swallowing, and symptoms were self-reported by the patient. This definition of dysphagia is largely consistent with others found in recently published literature. Incidences of dysphagia in the current study were 
quantified if symptoms were unresolved by the 2 week postoperative visit or if hospital readmission and additional treatment was required. Patients exhibiting symptoms past 2 weeks, but were resolved by 3 months without additional intervention or readmission, were considered to have "prolonged" dysphagia. Patients with symptoms unresolved after 3 months were considered to have "persistent" dysphagia.

Early complaints of postoperative, transient dysphagia were not uncommon in patients in the current series, but were not quantified prior to the initial postoperative visit at two weeks. Twenty-one (29\%) patients had prolonged dysphagia beyond the two-week postoperative visit, with symptoms resolving by 4 weeks in 10 patients and by 3 months in the remaining 11 patients. Of these, 2 patients required hospital readmission for observation and steroid treatment, with no additional intervention. No patients had persistent dysphagia after 3 months. By comparison, a retrospective analysis of 454 patients by Riley et al. found that the incidence of dysphagia at 3 months postoperative was $33 \%$ for patients treated at two levels and $39 \%$ for patients treated at three or more levels. $^{26}$

Excluding patients with uncomplicated prolonged dysphagia, the overall complication rate for patients in the current series was $11 \%$ (4\% major complications and $7 \%$ minor complications). By comparison, complication rates reported by Fountas et al. for two- or three-level ACDFs using autologous or allografts with or without a plate was $20 \%$. However, this complication rate decreases to $9 \%$ after excluding patients with dysphagia that resolved by 4 weeks postoperative. In their series, a postoperative hematoma occurred in $26(4.8 \%)$ patients, recurrent laryngeal nerve injury occurred in $18(3.3 \%)$ patients, and esophageal perforation occurred in $2(0.4 \%)$ patients. A CSF leak due to an intraoperative dural tear was noted in $2(0.4 \%)$ patients. $^{24}$

The fusion rate for patients in the current series was $98.6 \%$, or $99.5 \%$ of treated levels. By comparison, for patients treated with allograft or autograft, Fountas et al. reported similarly high fusion rates of $94 \%$ in two-level cases and $91 \%$ in three-level cases, giving an overall fusion rate of $93 \%$. A meta-analysis by Fraser et al. reported an $80 \%$ fusion rate for two-level ACDFs without fixation and $95 \%$ fusion rate with fixation. For three-level cases, Fraser et al. reported a $65 \%$ fusion rate for three-level cases without fixation and $83 \%$ fusion rate with additional plate placement. ${ }^{25} \mathrm{~A}$ more recent systematic literature review by Miller et al. reported fusion rates of $91 \%$ of levels treated for each allograft and autograft and $97 \%$ of levels treated with cages filled with morselized autologous local bone supplemented with bone graft substitute. ${ }^{28}$

Our fusion rate of $98.6 \%$ in the current study is also consistent with other rates reported for patients treated with BCS. Park et al. reported a $95.2 \%$ fusion rate in a series of 26 patients with BCS for one- or two-level degenerative disc disease. Of the 26 patients, allograft was used for 25 patients and autologous ICBG was used for 1 patient. ${ }^{27}$. In a comprehensive literature review, Vaccaro et al. also reported similarly high fusion rates of $94 \%$ of levels treated with BCS, and noted that sagittal alignment was maintained throughout the follow-up period. ${ }^{6}$ In comparison with other fusions rates for BCS with rhBMP-2, Lanman et al. reported a 100\% fusion rate by 3 months postoperative measured by $\mathrm{x}$-rays and CT. Low complication rates were also reported, with only 1 patient experiencing severe dysphagia requiring extended hospital stay. ${ }^{1}$ 
In the current series, BCS were used in conjunction with an average of $0.6 \mathrm{mg}$ of rhBMP-2 per level. While the authors recognize that the use of rhBMP-2 in the cervical spine is both off-label and controversial, we nonetheless hope the current study demonstrates that when used in very low doses and contained within a spacer and with meticulous surgical technique, rhBMP-2 can still be considered a safe and effective option. Particularly in light of recent findings from meta-reviews by Simmonds et al., the use of rhBMP-2 has been suggested to increase fusion rates when properly utilized. ${ }^{10}$

Clinical improvements in the current series were high, with an average of $60 \%$ reduction of neck pain and 55\% reduction of arm pain. Physical quality of life (QOL) improved an average of $24 \%$ and mental QOL improved an average of $18 \%$. Additionally, disability (NDI) improved an average of $43 \%$. In comparison, a systematic literature review by Miller et al. reported improvements in NDI as $46 \%$ for cage, $61 \%$ for allograft, and $76 \%$

for autograft. ${ }^{28}$ It should be noted that the clinical indication for surgery was myelopathy in $40 \%$ of patients in the current series, and thus, the NDI, which contains questions mostly related to disability as a result of radicular symptoms, may not reflect the "true disability" for these patients. Improvements for neck pain were reported between $63 \%$ to $69 \%$ and arm pain was reported as $62 \%$ to $75 \%$. Finally, PCS improved $42 \%$ and MCS improved $16 \%$ for patients treated with allograft.

Finally, there are several limitations in the current study, namely the lack of a control or comparative group, and the lack of routinely-collected CT scans. As this is an observational single-cohort study, conclusions regarding comparative effectiveness against conventional cage options are limited. Additionally, without an adequate control group, it is difficult to attribute clinical improvements and/or fusion efficacy to a specific component (interbody cage, biologic, etc.). The purpose of this study, instead, is to provide evidence regarding the feasibility and utility of a possible cage and graft combination. Finally, as serial CT studies were not collected, fusion status could only be determined based on symptomatic rather than radiographic confirmation.

\section{Conclusion}

Multilevel ACDF can be a highly effective treatment for cervical radiculopathy and myelopathy, although complications rates, particularly dysphagia, can be high, as can pseudoarthrosis rates. The results of this study suggest that proper use and dosage of rhBMP-2 contained within a bioabsorbable spacer, may facilitate high fusion rates while maintaining low complication rates and significant clinical improvements. Additionally, since bioabsorbable spacers gradually resorb, leaving no foreign implant in the intervertebral disc place, long-term findings of bridging bone makes fusion assessment more straightforward. Although the use of rhBMP-2 is both off-label and controversial, the results of the current study suggest that the combination of low-dose rhBMP-2 with BCS may be a useful treatment option with acceptable complication rates, high fusion rates, and good clinical improvements in patients undergoing multi-level ACDF.

\section{References}


1. Lanman TH, Hopkins TJ. Early findings in a pilot study of anterior cervical interbody fusion in which recombinant human bone morphogenetic protein-2 was used with poly(L-lactide-co-D,L-lactide) bioabsorbable implants. Neurosurg Focus 2004;16:6.

2. Malloy KM, Hilibrand AS. Autograft versus allograft in degenerative cervical disease. Clin Orthop 2002;394:27-38.

3. Bolesta MJ, Rechtine GR, 2nd, Chrin AM: One- and two-level anterior cervical discectomy and fusion: the effect of plate fixation. Spine J 2:197-203, 2002

4. Alexander JT, Branch CL, Jr., Subach BR, Haid RW: Applications of a resorbable interbody spacer via a posterior lumbar interbody fusion technique. Orthopedics 25:s1185-1189; discussion s1189, 2002

5. Toth JM, Estes BT, Wang M, Seim HB, Seifert JL, Turner AS, Cornwall GB: Evaluation of 70/30 poly (L-lactide-co-D,L-lactide) for use as a resorbable interbody fusion cage. J Neurosurg 97:423-432, 2002

6. Vaccaro AR, Singh K, Haid R, Kitchel S, Wuismann P, Taylor W, Branch C, Garfin S: The use of bioabsorbable implants in the spine. Spine J 3:227-237, 2003

7. Vaccaro AR, Venger BH, Kelleher PM, Singh K, Carrino JA, Albert T, Hilibrand A: Use of a bioabsorbable anterior cervical plate in the treatment of cervical degenerative and traumatic disk disruption. Orthopedics 25:s1191-1199; discussion s1199, 2002

8. Assietti R, Beretta F, Arienta C: Two-level anterior cervical discectomy and cageassisted fusion without plates. Neurosurg Focus 12:E3, 2002

9. Austin RC, Branch CL, Jr., Alexander JT: Novel bioabsorbable interbody fusion spacer-assisted fusion for correction of spinal deformity. Neurosurg Focus 14:e11, 2003

10. Simmonds MC, Brown JV, Heirs MK. Safety and effectiveness of recombinant human bone morphogenetic protein-2 for spinal fusion: a meta-analysis of individualparticipant data. Ann Intern Med 2013;158:877-89.

11. YODA 2

12. Lippman CR, Hajjar M, Abshire B, Martin G, Engelman RW, Cahill DW: Cervical spine fusion with bioabsorbable cages. Neurosurg Focus 16:E4, 2004

13. Bolesta MJ, Rechtine GR, 2nd, Chrin AM: Three- and four-level anterior cervical discectomy and fusion with plate fixation: a prospective study. Spine 25:2040-2044; discussion 2045-2046, 2000

14. Bose B: Anterior cervical instrumentation enhances fusion rates in multilevel reconstruction in smokers. J Spinal Disord 14:3-9, 2001

15. Cahill DW, Martin GJ, Jr., Hajjar MV, Sonstein W, Graham LB, Engelman RW: Suitability of bioresorbable cages for anterior cervical fusion. J Neurosurg 98:195-201, 2003

16. Cauthen JC, Theis RP, Allen AT: Anterior cervical fusion: a comparison of cage, dowel and dowel-plate constructs. Spine J 3:106-117; discussion 117, 2003

17. Coe JD: Instrumented transforaminal lumbar interbody fusion with bioabsorbable polymer implants and iliac crest autograft. Neurosurg Focus 16:E11, 2004

18. Cohen SR, Holmes RE, Amis P, Fichtner H: Internal craniofacial distraction with biodegradable devices: early stabilization and protected bone regeneration. $\mathrm{J}$ Craniofac Surg 11:354-366, 2000

19. Cornwall GB, Ames CP, Crawford NR, Chamberlain RH, Rubino AM: In vivo evaluation of bioresorbable polylactide implants for cervical graft containment in an ovine spinal fusion model. Neurosurg Focus 16:E5, 2004 
20. Couture DE, Branch CL, Jr.: Posterior lumbar interbody fusion with bioabsorbable spacers and local autograft in a series of 27 patients. Neurosurg Focus 16:E8, 2004

21. Floyd T, Ohnmeiss D: A meta-analysis of autograft versus allograft in anterior cervical fusion. Eur Spine J 9:398-403, 2000

22. Hwang SL, Lin CL, Lieu AS, Lee KS, Kuo TH, Hwang YF, Su YF, Howng SL: Three-level and four-level anterior cervical discectomies and titanium cageaugmented fusion with and without plate fixation. J Neurosurg Spine 1:160-167, 2004

23. Kuklo TR, Rosner MK, Polly DW, Jr.: Computerized tomography evaluation of a resorbable implant after transforaminal lumbar interbody fusion. Neurosurg Focus 16:E10, 2004

24. Fountas KN, Kapsalaki EZ, Nikolakakos LG, et al. Anterior cervical discectomy and fusion associated complication. Spine 2007;32:2310-7.

25. Fraser JF, Harti R. Anterior approaches to fusion of the cervical spine: a metaanalysis of fusion rates. J Neurosurg Spine 2007;6:298-303.

26. Riley LH, Skolasky RL, Albert TJ. Dysphagia after anterior cervical decompression and fusion. Spine 2005;30:2564-9.

27. Park MS, Aryan HE, Ozgur BM, et al. Stabilization of anterior cervical spne with bioabsorbable polymer in one- and two-level fusions. Neurosurg 2004;54:631-5.

28. Miller LE, Block JE. Safety and effectiveness of bone allografts in anterior cervical discectomy and fusion surgery. Spine 2011;36:2045-50.

\section{Disclosures}

The authors have declared no relevant financial disclosures.

Copyright (C) 2014 ISASS - International Society for the Advancement of Spine Surgery. To see more or order reprints or permissions, see http://ijssurgery.com. 